\title{
Gelation of Lyotropic Liquid-Crystal Phases-The Interplay between Liquid Crystalline Order and Physical Gel Formation
}

Sonja Dieterich, Thomas Sottmann, Frank Giesselmann*

Institute of Physical Chemistry, University of Stuttgart, Pfaffenwaldring 55, 70569 Stuttgart, Germany

\section{Supporting Information:}

Freeze fracture electron microscopy images
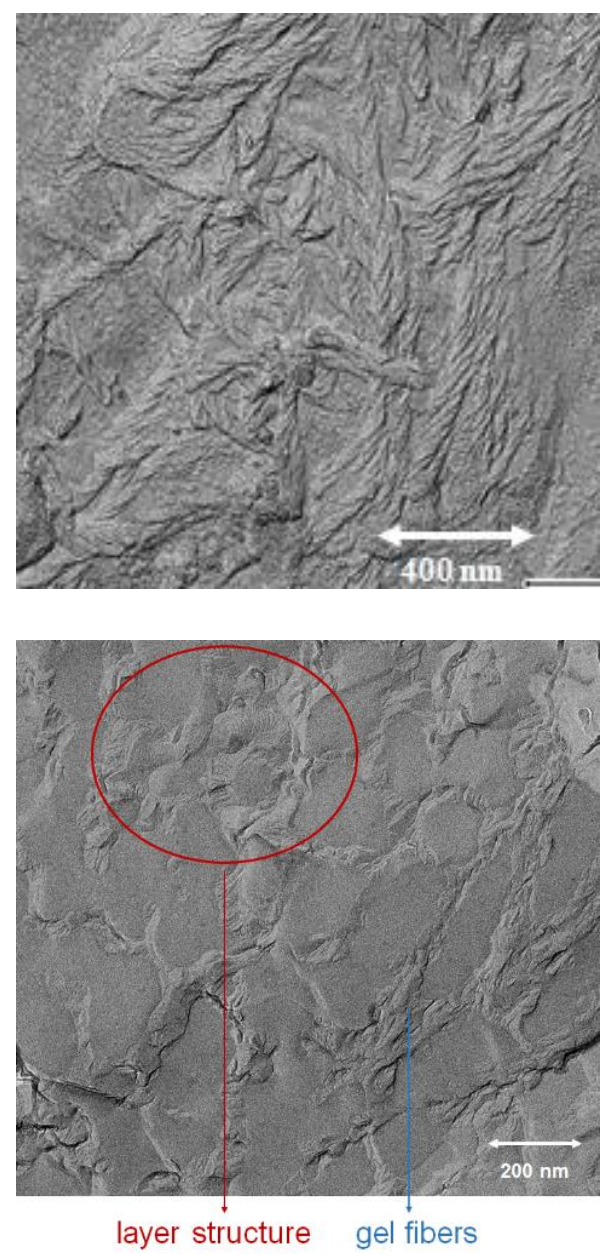

Figure S1: (top) Freeze fracture electron microscopy image of $n$-dodecane gelled with 3 wt.\% 12-HOA. The binary gels shows twisted gel fibers. (below) Freeze fracture electron microscopy image of the $\mathrm{N}_{\mathrm{c}}$ phase gelled with $3 \mathrm{wt} . \%$ 12-HOA. The observable layer steps demonstrate that gelling the $N_{c}$ phase leads to a gelled lamellar phase, where the layer structure coexists with twisted gel fibers. 